\title{
Sodium aescinate ameliorates liver injury induced by methyl parathion in rats
}

\author{
YUAN DU, TIAN WANG, NA JIANG, RU-TONG REN, CHONG LI, CHANG-KUN LI and FENG-HUA FU \\ Department of Pharmacology, School of Pharmacy, Yantai University, Yantai, Shandong 264005, P.R. China
}

Received November 18, 2011; Accepted January 6, 2012

DOI: 10.3892/etm.2012.479

\begin{abstract}
Methyl parathion, a highly cytotoxic insecticide, has been used in agricultural pest control for several years. The present study investigated the protective effect of sodium aescinate (SA, the sodium salt of aescin) against liver injury induced by methyl parathion. Forty male Sprague-Dawley rats were randomly divided into 5 groups of 8 animals: the control group; the methyl parathion $(15 \mathrm{mg} / \mathrm{kg})$ poisoning (MP) group; and the MP plus SA at doses of 0.45, 0.9 and $1.8 \mathrm{mg} / \mathrm{kg}$ groups. Alanine aminotransferase (ALT), aspartate aminotransferase (AST) and acetylcholinesterase (AChE) in the plasma were assayed. Nitric oxide (NO) and antioxidative parameters were measured. Histopathological examination of the liver was also performed. The results revealed that SA had no effect on AChE. Treatment with SA decreased the activities of ALT and AST, and the levels of malondialdehyde and NO. Treatment with SA also increased the level of glutathione and the activities of superoxide dismutase and glutathione peroxidase. SA administration also ameliorated liver injury induced by methyl parathion poisoning. The findings indicate that SA protects against liver injury induced by methyl parathion and that the mechanism of action is related to the antioxidative and anti-inflammatory effects of SA.
\end{abstract}

\section{Introduction}

Organophosphate compounds are the most extensively used insecticides. Millions of cases of organophosphate poisoning are reported annually worldwide, with the majority due to insecticide exposure. The widespread use and easy accessibility of these compounds result in a huge number of poisoning cases (1). Methyl parathion $(O-O$-dimethyl- $O$-p-nitrophenyl phosphorothioate), an organophosphorus compound that may only be lawfully used as an insecticide for agricultural crops, has recently received attention as a consequence of its illegal

Correspondence to: Professor Feng-Hua Fu, Department of Pharmacology, School of Pharmacy, Yantai University, 32 Qingquan Road, Yantai, Shandong 264005, P.R. China

E-mail: fufenghua@sohu.com

Key words: sodium aescinate, methyl parathion, liver injury, acute poisoning use (2). Tissue damage as a consequence of organophosphate poisoning is frequently reported; however, prevention of this potentially severe complication has not been the subject of considerable research. Particularly, hepatic dysfunction secondary to organophosphate exposure was reported in animals a few years ago (3).

Mechanisms other than AChE inhibition may be involved in the progression of acute organophosphate poisoning, including oxidative stress, mitochondrial energy metabolism impairment, microcirculation disturbance (4) and acute inflammation (5). Aesculus hippocastanum (Hippocastanaceae) is a plant that is distributed worldwide due to its excellent resistance to environmental conditions (6). Aescin, the major active agent from Aesculus hippocastanum, has recently been used in clinical therapy due to its anti-inflammatory and antioxidative effects. However, few studies have examined the effect of aescin on liver injury induced by phosphate pesticides. This study evaluates the effect of sodium aescinate on liver injury induced by methyl parathion poisoning.

\section{Materials and methods}

Chemicals. Methyl parathion (80\%, w/w) was obtained from Shandong Dacheng Co., Ltd (Zibo, China). Sodium aescinate (SA) was supplied by Shandong Luye Pharmaceutical Co., Ltd. (Yantai, China). Acetylcholinesterase (AChE), aspartate aminotransferase (AST), alanine aminotransferase (ALT), nitric oxide (NO), superoxide dismutase (SOD), glutathione peroxidase (GSH-Px), glutathione (GSH), malondialdehyde (MDA) and protein level test kits were purchased from the Institute of Jiancheng Bioengineering (Nanjing, China). All other chemicals and reagents used in this study were of analytical grade.

Animals and treatments. A total of 40 male Sprague-Dawley rats weighing $220 \pm 20 \mathrm{~g}$ were provided by the Experimental Animal Center of Shandong Engineering Research Center for Natural Drugs (Yantai, China), and the certificate number was 20030020. All experimental procedures conducted in this study were performed in accordance with the Guidelines for the Care and Use of Laboratory Animals of Yantai University. The rats were provided with free access to food and water on a 12-h light/dark cycle. They were housed in plastic cages and randomly divided into 5 groups of 8 animals: the control group; the methyl parathion $(15 \mathrm{mg} / \mathrm{kg}$ ) poisoning (MP) group; and the MP plus SA at doses of $0.45,0.9$ and $1.8 \mathrm{mg} / \mathrm{kg}$ groups. 
Rats received methyl parathion intragastrically to establish the acute methyl parathion poisoning model. The animals in the MP and SA groups were treated with SA via the tail vein at $2.5 \mathrm{~h}$ following methyl parathion poisoning, while the animals in the other groups were treated with normal saline in equivalent volumes. All animals were anesthetized with chloral hydrate $(300 \mathrm{mg} / \mathrm{kg}$, i.p.) $24 \mathrm{~h}$ following methyl parathion poisoning. A total of $5 \mathrm{ml}$ heparinized blood (1\% heparin, $100 \mu \mathrm{l}$ ) was collected from the abdominal aorta, and then the animals were sacrificed under anesthesia as a result of blood loss. The livers were excised and immediately cut into two, and subsequently washed with chilled normal saline. One section was fixed in paraformaldehyde (4\%, diluted in $0.1 \mathrm{~mol} / \mathrm{l}$ phosphate buffer solution, $\mathrm{pH}$ 7.4). The other section was weighed and homogenated.

Histopathological investigation. Paraformaldehyde-fixed, paraffin-embedded liver samples were cut into 4- $\mu \mathrm{m}$ sections, deparaffinized in xylene, and rehydrated through a series of descending concentrations of ethanol. Sections were stained with hematoxylin and eosin. Pathological observation of the tissues was performed under light microscopy.

Biochemical analysis. Blood samples were drawn into heparinized tubes for biochemical analysis. Following immediate centrifugation $\left(2500 \mathrm{x} \mathrm{g}\right.$ for $10 \mathrm{~min}$ at $\left.4^{\circ} \mathrm{C}\right)$, the plasma was stored at $-80^{\circ} \mathrm{C}$ until biochemical analysis. The livers were weighed and homogenized in ice-cold normal saline (1/9, $\mathrm{w} / \mathrm{v})$ at a speed of $5000 \mathrm{rpm}(15 \mathrm{sec} \times \mathrm{5})$. The suspension was centrifuged at a speed of $2500 \mathrm{x}$ g for $10 \mathrm{~min}$ at $4^{\circ} \mathrm{C}$, and the supernatant was stored at $-80^{\circ} \mathrm{C}$. The activities of AChE, ALT, AST, SOD and GSH-Px, and the levels of NO, GSH and MDA were determined, respectively, according to the manufacturer's instructions.

Statistical analysis. The one-way ANOVA test was used to analyze the significant differences between the different groups. Comparisons between two groups were determined using the Student's unpaired t-test, using SPSS 11.5 statistical software. $\mathrm{P}<0.05$ was considered to indicate a statistically significant difference. All data in the study are expressed as the means \pm SD.

\section{Results}

Effect of SA on the pathological changes in rat livers. Normal architecture of the liver was observed in the control group (Fig. 1A). Inflammatory cell clusters, severe congestion of the hepatic sinusoids, hepatocyte necrosis and steatosis were observed in the MP group (Fig. 1B). SA significantly ameliorated the pathological changes induced by methyl parathion poisoning (Fig. 1C-E).

Effect of SA on AChE activity in the plasma following methyl parathion poisoning. The AChE activity was significantly inhibited following MP administration. SA had no effect on the reduction of AChE activity (Fig. 2).

Effect of SA on ALT and AST activities in the plasma following methyl parathion poisoning. The activities of ALT
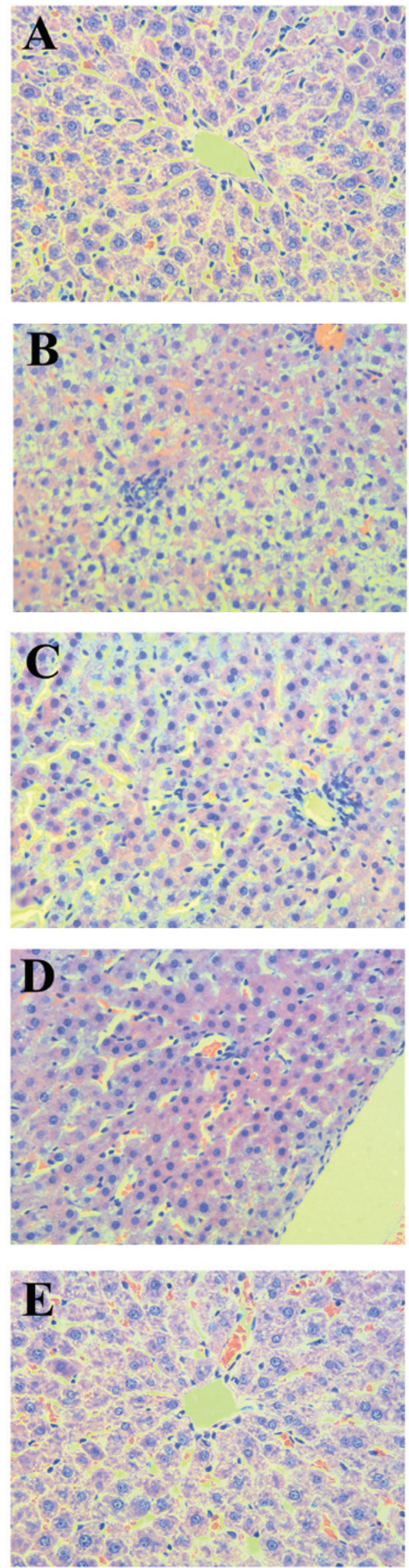

Figure 1. (A) Microscopic appearance of liver tissue in the (A) control group and (B) MP group (magnification, x400). Microscopic appearance of liver tissues in the (C) MP and $0.45 \mathrm{mg} / \mathrm{kg} \mathrm{SA}$ group, (D) $\mathrm{MP}$ and $0.9 \mathrm{mg} / \mathrm{kg} \mathrm{SA}$ group, and (E) MP and $1.8 \mathrm{mg} / \mathrm{kg} \mathrm{SA}$ group (magnification, $\mathrm{x} 400$ ). MP, methyl parathion $(15 \mathrm{mg} / \mathrm{kg}$ ) poisoning; SA, sodium aescinate. 


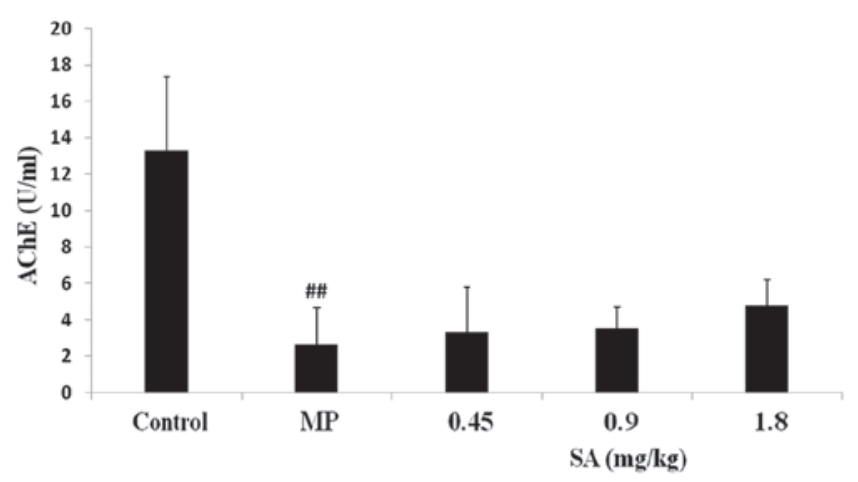

Figure 2. Effect of SA on AChE activity in the plasma. The SA treatment groups were administered SA at doses of $0.45,0.9$ and $1.8 \mathrm{mg} / \mathrm{kg}$. The control and MP group were administered normal saline. Data are expressed as the means $\pm \mathrm{SD}(\mathrm{n}=7-8)$. ${ }^{\# \#} \mathrm{p}<0.01$ compared with the control group. AChE, acetylcholinesterase; MP, methyl parathion $(15 \mathrm{mg} / \mathrm{kg})$ poisoning; SA, sodium aescinate.

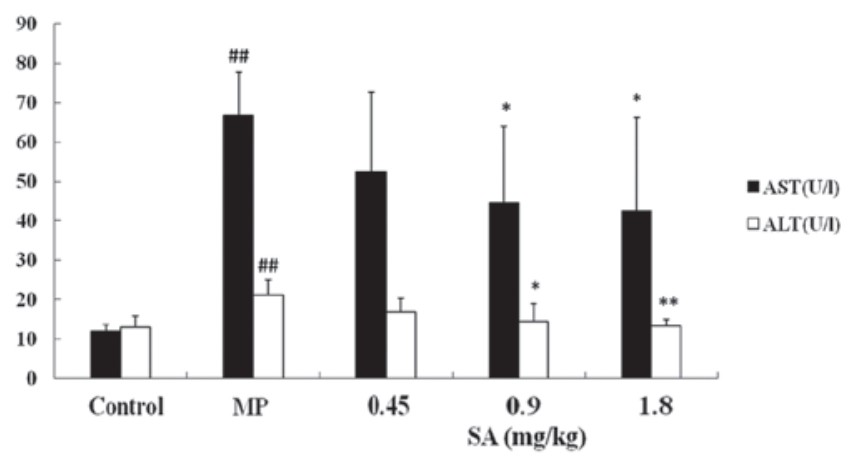

Figure 3. Effect of SA on ALT and AST activities in the plasma. The SA treatment groups were administered SA at doses of $0.45,0.9$ and $1.8 \mathrm{mg} / \mathrm{kg}$. The control and MP group were administered normal saline. Data are expressed as the means $\pm \mathrm{SD}(\mathrm{n}=7-8) .{ }^{\#} \mathrm{p}<0.01$ compared with the control group; ${ }^{*} \mathrm{p}<0.05 ;{ }^{* *} \mathrm{p}<0.01$ compared with the MP group. MP, methyl parathion $(15 \mathrm{mg} / \mathrm{kg})$ poisoning; SA, sodium aescinate; AST, aspartate aminotransferase; ALT, alanine aminotransferase.

and AST increased markedly following MP poisoning. SA $(0.9$ and $1.8 \mathrm{mg} / \mathrm{kg}$ ) treatment decreased ALT and AST activities (Fig. 3).

Effect of SA on NO level in the plasma and liver following methyl parathion poisoning. Compared with the control group, the NO content in the MP group significantly increased. SA suppressed the elevation of the NO level in the plasma and liver tissue (Figs. 4 and 5).

Effect of SA on SOD, GSH-Px activities and GSH, MDA levels in the liver following methyl parathion poisoning. Analysis of the antioxidative parameters revealed that administration of methyl parathion resulted in a significant decrease in the activities of SOD and GSH-Px, and the level of GSH. Furthermore, methyl parathion poisoning also led to an increase in the level of MDA. However, treatment with SA attenuated the changes in the activities of SOD and GSH-Px, and the levels of GSH and MDA (Figs. 6-9).

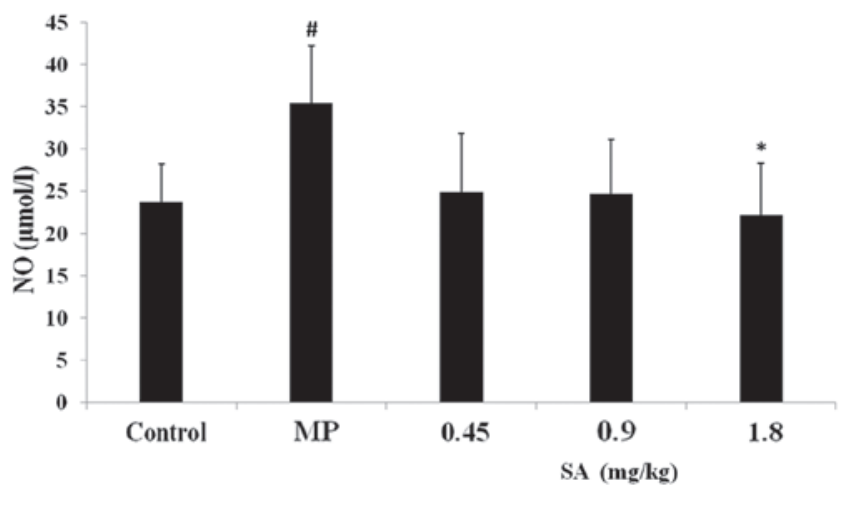

Figure 4. Effect of SA on NO level in the plasma. The SA treatment groups were administered SA at doses of $0.45,0.9$ and $1.8 \mathrm{mg} / \mathrm{kg}$. The control and MP group were administered normal saline. Data are expressed as the means $\pm \mathrm{SD}(\mathrm{n}=7-8) .{ }^{*} \mathrm{p}<0.05$ compared with the control group; ${ }^{*} \mathrm{p}<0.05$ compared with the MP group. NO, nitric oxide; MP, methyl parathion $(15 \mathrm{mg} / \mathrm{kg})$ poisoning; SA, sodium aescinate.

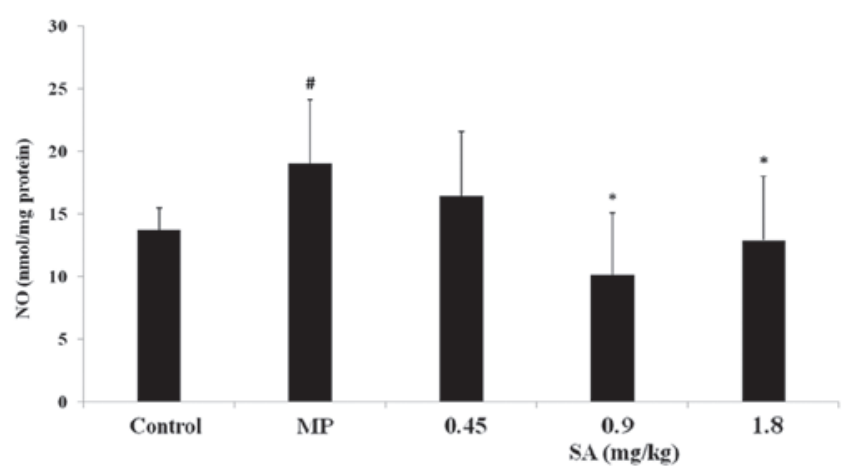

Figure 5. Effect of SA on NO level in the liver. The SA treatment groups were administered SA at doses of $0.45,0.9$ and $1.8 \mathrm{mg} / \mathrm{kg}$. The control and MP group were administered normal saline. Data are expressed as the means $\pm \mathrm{SD}(\mathrm{n}=7-8) .{ }^{*} \mathrm{p}<0.05$ compared with the control group; ${ }^{*} \mathrm{p}<0.05$ compared with the MP group. NO, nitric oxide; MP, methyl parathion $(15 \mathrm{mg} / \mathrm{kg})$ poisoning; SA, sodium aescinate.

\section{Discussion}

The liver plays a pivotal role in a large number of metabolic and immune processes; therefore, the hepatotoxicity of the liver as a result of toxic agents, and the potential therapeutic strategies have attracted numerous studies. The systems and organs that can be influenced by organophosphate intoxicants are the immune, urinary and reproductive systems, the pancreas, liver and the lungs. Certain studies have reported that organophosphates cause liver damage (7). ALT and AST are significant indicators of liver damage. These enzymes were revealed to leak out into the blood following hepatocellular injury (3). Furthermore, other studies have also indicated that organophosphates lead to serious changes in hepatocytes and organelles; for example, an increase in the chromatin content of hepatocyte nuclei and cytoplasmic density. The involved cells also became vacuolar in appearance as a result of lysis in the mitochondrial matrices. In certain cells, the lipid content constituted the majority of the cytoplasm. It was also revealed that collagen fibers expand to form bands in certain areas of the 


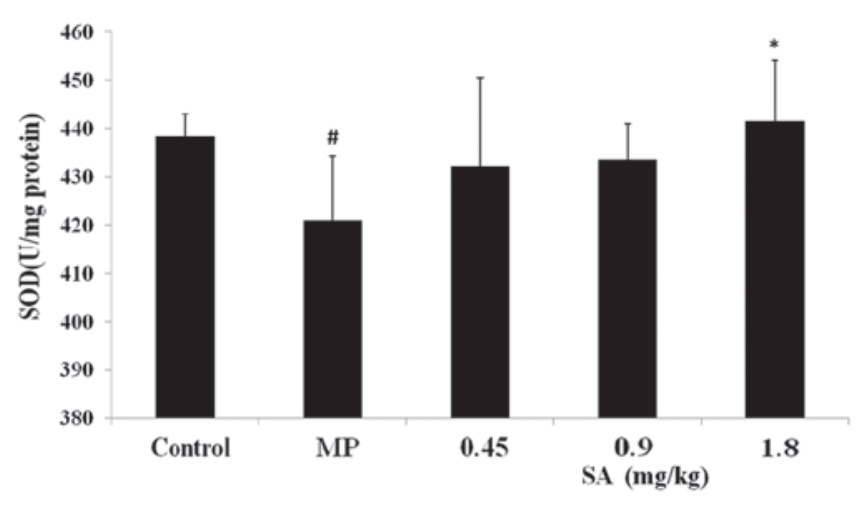

Figure 6. Effect of SA on SOD activity in the liver. The SA treatment groups were administered SA at doses of $0.45,0.9$ and $1.8 \mathrm{mg} / \mathrm{kg}$. The control and MP group were administered normal saline. Data are expressed as the means $\pm \mathrm{SD}(\mathrm{n}=7-8) .{ }^{*} \mathrm{p}<0.05$ compared with the control group; ${ }^{*} \mathrm{p}<0.05 \mathrm{com}-$ pared with the MP group. SOD, superoxide dismutase; MP, methyl parathion $(15 \mathrm{mg} / \mathrm{kg})$ poisoning; SA, sodium aescinate.

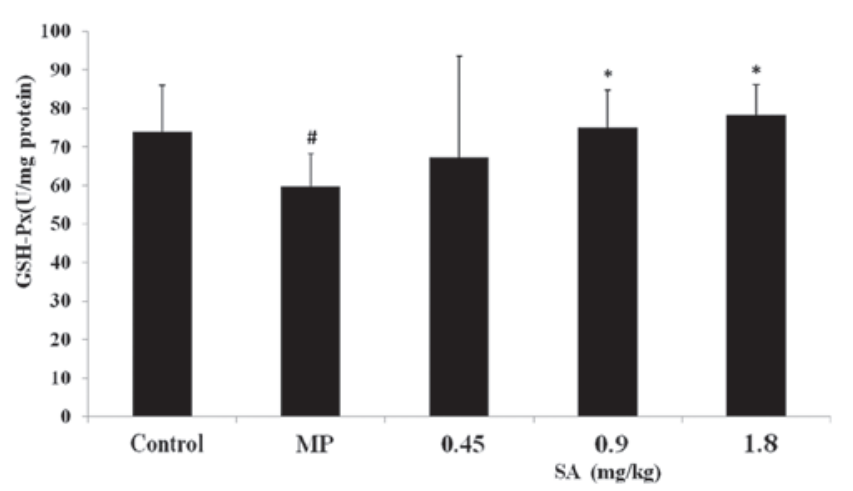

Figure 7. Effect of SA on GSH-Px activity in the liver. The SA treatment groups were administered SA at doses of $0.45,0.9$ and $1.8 \mathrm{mg} / \mathrm{kg}$. The control and MP group were administered normal saline. Data are expressed as the means $\pm \mathrm{SD}$ $(\mathrm{n}=7-8) .{ }^{*} \mathrm{p}<0.05$ compared with the control group; ${ }^{*} \mathrm{p}<0.05$ compared with the MP group. GSH-Px, glutathione peroxidase; MP, methyl parathion $(15 \mathrm{mg} / \mathrm{kg})$ poisoning; $\mathrm{SA}$, sodium aescinate.

liver (8). In our study, MP caused a significant increase in the activities of the ALT and AST enzymes. Histological damage in the MP-treated rats was also observed. However, SA treatment markedly reduced the MP-induced hepatic dysfunction, as revealed by a significant reduction in the serum ALT and AST enzyme activities, and an attenuation in the histological changes in the liver.

NO is a key factor in hepatic injury (9). Increased levels of $\mathrm{NO}$ are a natural sequence to the inhibition of AChE by organophosphates. A number of studies have demonstrated that NO may promote inflammation-induced cell and tissue dysfunction. NO-dependent reactions are significant in modulating the inflammatory response and may account for hepatic necrosis through specific signaling mechanisms $(10,11)$. The liver is an organ that is evidently influenced by NO. Therefore, when a large sustained amount of $\mathrm{NO}$ is present, damage occurs in the liver (12). Otherwise, the serious liver injury may induce excessive systemic inflammation (13). In the present study, we measured the level of $\mathrm{NO}$ in plasma and liver tissue. Our

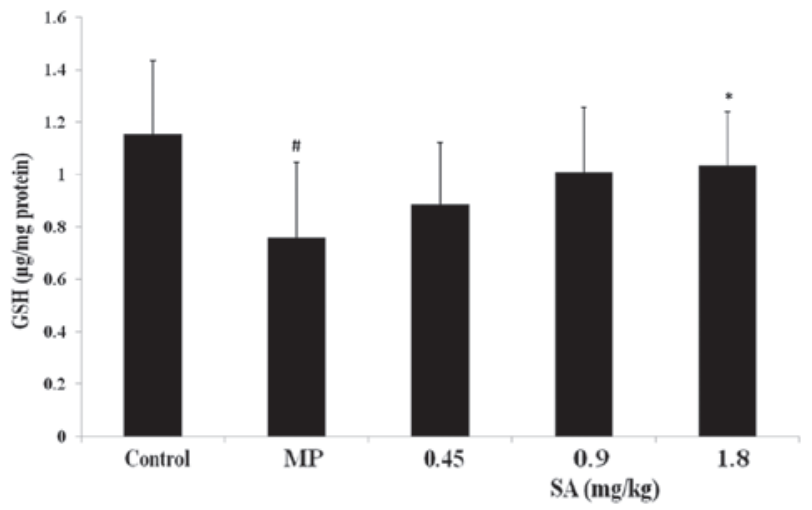

Figure 8. Effect of SA on GSH level in the liver. The SA treatment groups were administered SA at doses of $0.45,0.9$ and $1.8 \mathrm{mg} / \mathrm{kg}$. The control and MP group were administered normal saline. Data are expressed as the means $\pm \mathrm{SD}(\mathrm{n}=7-8) .{ }^{\#} \mathrm{p}<0.05$ compared with the control group; ${ }^{*} \mathrm{p}<0.05$ compared with the MP group. GSH, glutathione; MP, methyl parathion $(15 \mathrm{mg} / \mathrm{kg})$ poisoning; SA, sodium aescinate.

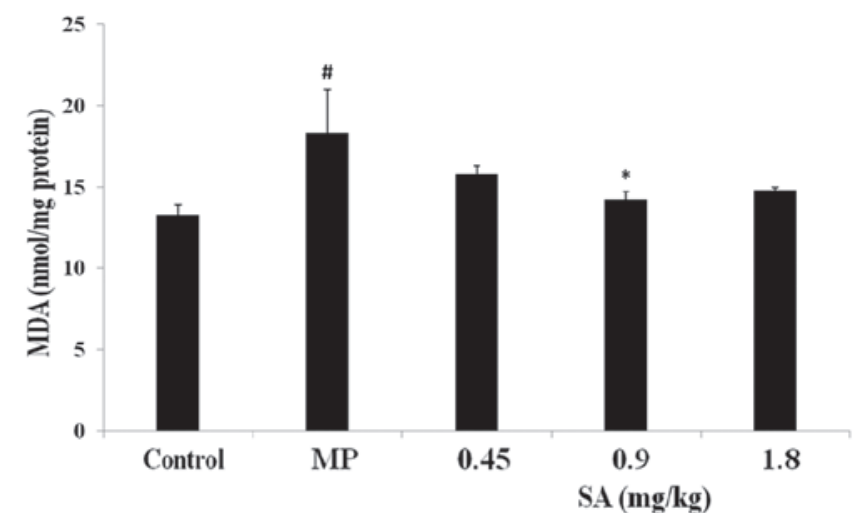

Figure 9. Effect of SA on MDA level in the liver. The SA treatment groups were administered SA at doses of $0.45,0.9$ and $1.8 \mathrm{mg} / \mathrm{kg}$. The control and MP group were administered normal saline. Data are expressed as the means $\pm \mathrm{SD}(\mathrm{n}=7-8) .{ }^{*} \mathrm{p}<0.05$ compared with the control group; ${ }^{*} \mathrm{p}<0.05$ compared with the MP group. MDA, malondialdehyde; MP, methyl parathion (15 mg/kg) poisoning; SA, sodium aescinate.

study demonstrated a significant increase in nitrate (measured as nitrite), the stable end product of NO, in the plasma and the liver in the MP-treated group. As expected, SA treatment significantly inhibited MP-induced NO production.

Pesticides have been reported to induce the generation of reactive oxygen species (ROS) in vitro and in vivo (14). ROS are significant in the toxicity of organophosphate compounds (15). Oxidative damage by free radicals or ROS could result in lipid peroxidation, causing changes in membrane properties and cell dysfunction (16). Organophosphate pesticides may induce oxidative stress, leading to the generation of free radicals and an alteration in antioxidants (17). It was revealed that the lipid peroxidative substance (MDA) was elevated and SOD was reduced following organophosphate poisoning (4). Methyl parathion was also able to deplete GSH in the rat liver by forming GSH conjugates (18). In addition, the activity of GSH-Px was diminished following organophosphate poisoning (19). Our study revealed that organophosphate poisoning resulted in a change in oxidative stress. However, SA administration 
ameliorated the oxidative damage. These findings are in accordance with a previous study which reported that SA improves the antioxidative defense system (20).

The results from the present study demonstrated that acute organophosphate poisoning causes serious histopathological changes in the rat liver; however, these changes are reversible following SA treatment. The pharmacological action of SA is associated with its antioxidative and anti-inflammation effects. The protective effects of SA on liver injury induced by organophosphates require further study.

\section{Acknowledgements}

The authors would like to thank Tongshen Liu for his technical assistance. This study was supported by the Taishan Scholar Project, the 11th Five-Year Key Programs for Science and Technology Development of China (grant no. 2008ZX09202-008) and the National Natural Science Foundation of China (grant no. 30772760).

\section{References}

1. Atiş S, Cömelekoğlu U, Coşkun B, Ozge A, Ersöz G and Talas D: Electrophysiological and histopathological evaluation of respiratory tract, diaphragm, and phrenic nerve after dichlorvos inhalation in rats. Inhal Toxicol 14: 199-215, 2002.

2. Zhu H, Rockhold RW, Baker RC, Kramer RE and Ho IK: Effects of single or repeated dermal exposure to methyl parathion on behavior and blood cholinesterase activity in rats. J Biomed Sci 8: 467-474, 2001.

3. Kalender S, Ogutcu A, Uzunhisarcikli M, Açikgoz F, Durak D, Ulusoy Y and Kalender Y: Diazinon-induced hepatotoxicity and protective effect of vitamin $\mathrm{E}$ on some biochemical indices and ultrastructural changes. Toxicology 211: 197-206, 2005.

4. Zhang X, Yao W, Jia B, Sun D, Ka W, He D, Wang X and Wen Z: Acute dichlorvos poisoning induces hemorheological abnormalities in rabbits via oxidative stress. Clin Hemorheol Microcirc 44: 207-216, 2010

5. Ouyang YH, Li SL, Song W, Zhao N and Ma ZF: Effect of penehyclidine hydrochloride on tumor necrosis factor-alpha in liver and spleen in mice with acute organophosphorus pesticide poisoning. Zhongguo Wei Zhong Bing Ji Jiu Yi Xue 22: 238-239, 2010 (In Chinese).
6. Sirtori CR: Aescin: pharmacology, pharmacokinetics and therapeutic profile. Pharmacol Res 44: 183-193, 2001.

7. Yurumez Y, Ikizceli I, Sozuer EM, Soyuer I, Yavuz Y, Avsarogullari L and Durukan P: Effect of interleukin-10 on tissue damage caused by organophosphate poisoning. Basic Clin Pharmacol Toxicol 100: 323-327, 2007.

8. Satar S, Satar D, Tap O, Koseoglu Z and Kaya M: Ultrastructural changes in rat liver treated with pralidoxime following acute organophosphate poisoning. Mt Sinai J Med 71: 405-410, 2004.

9. Senga F, Yin L, Karasuno H, Ohtaki H, Nakamachi T, Satoh K and Shioda S: Minus charge stimulation prevents LPS-induced liver injury by reduction of nitric oxide. J Clin Biochem Nutr 42: 222-227, 2008

10. Li J and Billiar TR: Nitric Oxide. IV. Determinants of nitric oxide protection and toxicity in liver. Am J Physiol 276: G1069-G1073, 1999.

11. Grisham MB, Jourd'Heuil D and Wink DA: Nitric oxide. I. Physiological chemistry of nitric oxide and its metabolites: implications in inflammation. Am J Physiol 276: 15-21, 1999.

12. Hon WM, Lee KH and Khoo HE: Nitric oxide in liver diseases: friend, foe, or just passerby? Ann NY Acad Sci 962: 275-295, 2002.

13. Johnson D and Mayers I: Multiple organ dysfunction syndrome: a narrative review. Can J Anaesth 48: 502-509, 2001.

14. Abdollahi M, Ranjbar A, Shadnia S, Nikfar S and Rezaie A: Pesticides and oxidative stress: a review. Med Sci Monit 10: 141-147, 2004.

15. Gunay N, Kose B, Demiryurek S, Ocak AR, Erel O and Demiryurek AT: Effects of a selective Rho-kinase inhibitor Y-27632 on oxidative stress parameters in acute dichlorvos poisoning in rats. Cell Biochem Funct 26: 747-754, 2008.

16. Sinclair AJ, Barnett AH and Lunec J: Free radicals and antioxidant systems in health and disease. Br J Hosp Med 43: 334-344, 1990

17. Agrawal D, Sultana P and Gupta GS: Oxidative damage and changes in the glutathione redox system in erythrocytes from rats treated with hexachlorocyclohexane. Food Chem Toxicol 29: 459-462, 1991

18. Della Morte R, Villani GR, Di Martino E, Squillacioti C, De Marco L, Vuotto P, Belisario MA and Staiano N: Glutathione depletion induced in rat liver fractions by seven pesticides. Boll Soc Ital Biol Sper 70: 185-192, 1994.

19. Khan SM, Sobti RC and Kataria L: Pesticide-induced alteration in mice hepato-oxidative status and protective effects of black tea extract. Clin Chim Acta 358: 131-138, 2005.

20. Küçükkurt I, Ince S, Keleş H, Akkol EK, Avci G, Yeşilada E and Bacak E: Beneficial effects of Aesculus hippocastanum L. seed extract on the body's own antioxidant defense system on subacute administration. J Ethnopharmacol 129: 18-22, 2010. 